\title{
The kinematics of 867 galactic planetary nebulae ${ }^{\star}$
}

\author{
S. Durand ${ }^{1}$, A. Acker ${ }^{1}$, and A. Zijlstra ${ }^{2}$ \\ 1 UMR 7550, Équipe Évolution Galactique, Observatoire de Strasbourg, 11 rue de l'Université, F-67000 Strasbourg, France \\ 2 UMIST, department of Physics, P.O. Box 88, Manchester M60 1QD, UK
}

Received April 18, 1997; accepted March 24, 1998

\begin{abstract}
We present a compilation of radial velocities of 867 galactic planetary nebulae. Almost 900 new measurements are included. Previously published kinematical data are compared with the new high-resolution data to assess their accuracies. One of the largest samples in the literature shows evidence for a systematic velocity offset. We calculate weighted averages between all available data. Of the final values in the catalogue, $90 \%$ have accuracies better than $20 \mathrm{~km} \mathrm{~s}^{-1}$. We use this compilation to derive kinematical parameters of the galactic differential rotation obtained from least-square fitting and to establish the Disk rotation curve; we find no significal trend for the presence of an increasing external rotation curve. We examine also the rotation of the bulge; the derived curve is consistent with a linearly increasing rotation velocity with $l$ : we find $V_{\mathrm{b}, \mathrm{r}}=(9.9 \pm 1.3) l-(6.7 \pm 8.5) \mathrm{km} \mathrm{s}^{-1}$. A possible steeper gradient in the innermost region is indicated.
\end{abstract}

Key words: catalogues — planetary nebulae — Galaxy (the): kinematics of

\section{Introduction}

Kinematical studies of the stellar populations in the inner parts of the Milky Way have mainly used RGB and AGB stars, observed in strategically chosen lowextinction windows (Morrison et al. 1990; Menzies 1990; Minniti 1994; Izumiura et al. 1995; Sevenster et al. 1997). These stars are both bright and numerous, and their metallicity covers a large range, allowing one to study the correlations between the kinematics and the chemical abundances in the Galaxy (Lewis \& Freeman 1989; Ibata \& Gilmore 1995). Recently, proper motions for some $\mathrm{K}$ giants have become available which provide the first

Send offprint requests to: S. Durand

* Table 2 is available in electronic form only, via anonymous ftp to cdsarc.u-strasbg.fr (130.79.128.5) or via http://cdsweb.u-strasbg.fr/Abstract.html striking signature of the bulge triaxiality (Zhao et al. 1994).

Planetary nebulae (hereafter $\mathrm{PNe}$ ) trace a closely related stellar population which can also be used for such studies. They originate from intermediate and low initial-mass stars and therefore constitute a relatively old population. The optical spectrum of a $\mathrm{PN}$ is dominated by bright emission lines [recombination ( $\mathrm{H}, \mathrm{He}$, a.o.) and collisionally excited ([OIII], a.o.) lines] allowing highly accurate radial velocity determinations. The observable life time is short, typically a few times $10^{4}$ years. Therefore PNe are not very numerous in the Galaxy: the total population in the Galactic disk is estimated to be around 23000 with an additional 2000 in the bulge (Zijlstra \& Pottasch 1991); older determinations range between 7000 and 70000 (see the compilation in Peimbert 1992) but with large dependencies on adopted distance scales. To date, about $1700 \mathrm{PNe}$ are known in the Galaxy, classified in the Strasbourg-ESO Catalogue (Acker et al. 1992, hereafter SECAT) and its first supplement (Acker et al. 1996).

Although this suggests that $\mathrm{PNe}$ are useful tracers of Galactic kinematics, so far few papers have directly studied the kinematics of the PNe population. Schneider et al. (1983a; hereafter STPP83) made a compilation of all known $\mathrm{PNe}$ radial velocities at that time, correcting for systematic offsets between different sources. In a follow-up paper, Schneider \& Terzian (1983b) derived a rising rotation curve in the outer Galaxy from 252 disk PNe. Kinman et al. (1988; hereafter KFL88) found evidence for bulge rotation from $\mathrm{PNe}$ radial velocities.

Since the compilation of STPP83, further radial velocity determinations of $\mathrm{PNe}$ have become available. Meatheringham et al. (1988; hereafter MWF88) presented radial and expansion velocities of 64 southern galactic planetary nebulae. In the same year KFL88 published radial velocities of $23 \mathrm{PNe}$ (although with low accuracy), 15 of which were newly discovered. More recently, Kohoutek 
\& Pauls (1995) presented well-determined radial velocities for 76 nebulae in the direction of the Galactic centre. Spyromilio (1995) gave accurate velocities for a few non-bulge PNe. Dopita \& Hua (1997) measured radial velocities and fluxes of 52 southern PNe. Beaulieu (1996) made a deep and uniform narrow-band $\mathrm{H} \alpha$ imaging survey for PNe in the southern Galactic bulge; she measured radial velocities of 56 newly discovered $\mathrm{PNe}$ and also re-determined velocities for 317 previously known $\mathrm{PNe}$. Finally, Zijlstra et al. (1997) presented high-accuracy velocities for 71 Galactic bulge PNe. There is significant overlap between these samples. Combined, there is now a larger and more accurate set of data available especially for the central regions of the Galaxy.

In this paper we present a new, up-to-date and homogeneous compilation of all PNe kinematical data known at the present time. A large number of new and previously unpublished measurements are included. The paper is presented as follows: Sect. 2 presents new radial velocities coming from our recent observations. In Sect. 3 the catalogue is presented, with a brief overview of its main characteristics. Finally, in Sect. 4, a preliminary study of the kinematics of the disk and the bulge is made.

\section{New radial velocities}

We have measured radial velocities of Galactic PNe in three separate observational programs. These concern 338 different $\mathrm{PNe}, 93$ of which have for the first time a measured radial velocity. Some of these data have been published (Zijlstra et al. 1997); the remainder is presented here. The three programs are:

- 117 high-resolution data $(Z A S W$, in part published in Zijlstra et al. 1997).

In the context of a study of expansion velocities of galactic bulge $\mathrm{PNe}$ and of $\mathrm{PNe}$ with [WC]-type nuclei, accurate radial velocities were measured for a sample of $119 \mathrm{PNe}$ (this sample we will designate $Z A S W$ ). The observations were carried out with the ESO $1.4 \mathrm{~m}$ CAT telescope with the short camera (CES), and performed in 1993 June, in 1993 July and in 1994 March. Two spectral ranges were selected, one around the [OIII] 5007 line ( $R=30$ 000, 1993 June), the other including $\mathrm{H} \alpha$ and the $[\mathrm{NII}]$ red doublet $(R=60000,1993$ July and 1994 March, see Acker 1993). Two PNe were discovered to belong to the Sagittarius Dwarf galaxy (Zijlstra \& Walsh 1996), and therefore are excluded from our Galactic sample. The observations and the radial velocities of 71 bulge $\mathrm{PNe}$ (labelled as $(z)$ in Table 2 (Col. 3) are presented in Zijlstra et al. 1997). The velocities of the 48 non-bulge PNe obtained from $\mathrm{H} \alpha$ and [NII] $(R=60000)$ measurements are given in Table 2 Col. 3 (labelled as $(a)$ ).

\section{- 130 medium-resolution data $(A S C)$.}

In the framework of chemical abundance determinations of galactic PNe high above the Galactic plane (Cuisinier et al. 1996), observations were conducted from 1993 to 1995 at the ESO $1.52 \mathrm{~m}$ telescope using the Boller \& Chivens spectrograph and the $2048 \times$ 2048 pixel FA27 CCD CCD. The wavelength range was $3600-7400 \AA$ with a spectral resolution $R \simeq 1500$. The entrance aperture was $1.5 \times 3.4$ arcsec.

- 247 low-resolution data $(S A)$.

From 1983 to 1991, a spectrophotometric survey of galactic PNe was performed at the ESO $1.52 \mathrm{~m}$ telescope equipped with the Boller \& Chivens spectrograph (see Acker et al. 1989b; Acker et al. 1992). Until 1987 the IDS detector was used, and afterwards a $512 \times 1024$ pixel CCD. The wavelength range was $3600-7400 \AA$ with a spectral resolution $R \simeq 800$. As the sample included a large number of Galactic bulge $\mathrm{PNe}$, we measured the radial velocities of those 247 $\mathrm{PNe}$ despite the low resolution of the spectra.

The $A S C$ and $S A$ sample showed wavelengthdependent velocity residuals. To remove these, we applied a linear fit to these deviations per observing run (the slope of the fit changed between observing runs). The values on the blue part of the spectra appeared to be of poor quality (probably due to the low sensitivity of the detector) and were removed from the calculations. Each run was calibrated using the available $Z A S W$ values. Considering the mean dispersions of the spectra, measurements for which the uncertainties exceeded the expected ones were not included in the catalogue.

From the medium-resolution $A S C$ data we obtained 130 radial velocities of sufficient quality, with a mean uncertainty of about $20 \mathrm{~km} \mathrm{~s}^{-1}$. From the $S A$ data we obtained 247 acceptable measurements with a mean uncertainty of about $40 \mathrm{~km} \mathrm{~s}^{-1}$.

The galactic distribution of these 494 new data (including data published in Zijlstra et al. 1997) is displayed in Fig. 1.

\section{The catalogue}

The catalogue contains:

- 494 new high-, medium- and low-resolution measurements described in Sect. 2.

- 373 nebulae from Beaulieu (1996), 73 from Kohoutek \& Pauls (1995), 52 from Dopita \& Hua (1997), 3 from Spyromilio (1995) and 5 from Kraan-Korteweg et al. (1996).

- 577 radial velocities published in the SECAT (including STPP83, MWF88 and KFL88 data). 


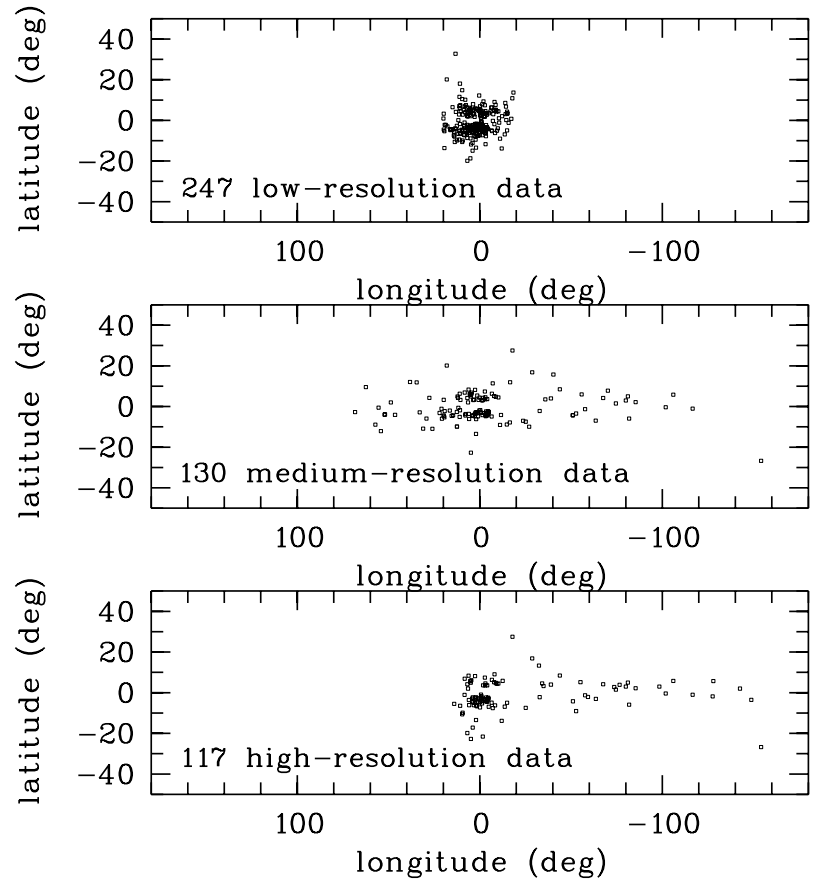

Fig. 1. Galactic positions of the new 494 radial velocities - top: $247 S A$ data; - middle: $130 A S C$ data; - bottom: $117 Z A S W$ data

\subsection{Uncertainties and systematic deviations of literature data}

The literature data originates mostly from a number of individual surveys. In order to test the accuracies of these surveys, we compare the lists with the high-resolution $Z A S W$ data, for those objects in common.

Figure 2 (right) displays the comparison of the literature data data with the high-resolution $Z A S W$ data for several surveys. Two individual objects of Dopita \& Hua, He $2-118$ and He $2-97$, show a very large discrepancy with the $Z A S W$ values (see Fig. 2, right) and are excluded in the statistics of Table 1. Both have late-type [WC] central stars, and in such objects a velocity shift is sometimes seen between nebular and stellar lines (e.g. Menzies \& Wolstencroft 1990; Le Bertre et al. 1989). Two large surveys included in STPP83 (Minkowski 1957 (right panel) and Mayall 1964 (left panel)) show significant scatter. Both are published only as private communications in the catalogue of Perek \& Kohoutek (1967) and may originate from a variety of separate measurements.

Table 1 presents the systematic deviations and the scatter for the principal sources. We calculate the significance of the systematic offsets between the samples by applying student's $t$ statistics on the objects in common between $Z A S W$ and the individual samples. An uncertainty of $2 \mathrm{kms}^{-1}$ is assigned to $Z A S W$ (Zijlstra et al. 1997). The resulting probabilities are listed in Table 1. The offset is taken to be significant if the probability is
Table 1. Systematic deviations of the principal sources of available data compared with the $Z A S W$ data ( $n$ is the number of compared PNe) (offsets stand for $\left.<V_{\mathrm{ZASW}}-V_{i}\right\rangle$ ). The probability gives the likelihood that the offset is due to chance, using student's $t$ statistics

\begin{tabular}{|l|ll|l|l|}
\hline Sources & offsets disp. & $n$ & prob. \\
\hline \hline AS (1992) & & & & \\
\hline ASC (1995) & -4.1 & 39.3 & 52 & 0.46 \\
\hline Campbell \& Moore (1918) & -2.7 & 18.4 & 55 & 0.29 \\
\hline Minkowski (1957) & -16.3 & 4.4 & 11 & 0.058 \\
\hline Mayall (1964) & -3.0 & 42.4 & 23 & 0.74 \\
\hline Acker (1975) & -6.4 & 9.6 & 08 & 0.1 \\
\hline Webster \& Kalnajs (1982) & -2.7 & 2.9 & 11 & 0.02 \\
\hline Kohoutek \& Pauls (1995) & +0.8 & 4.2 & 07 & 0.66 \\
\hline Dopita \& Hua (1997) & +4.3 & 8.3 & 10 & 0.14 \\
\hline Beaulieu (1996) & +4.1 & 15.2 & 50 & 0.064 \\
\hline
\end{tabular}

less than 0.01. This is the case only for Minkowski (1957). STPP83 added $4 \mathrm{kms}^{-1}$ to all Webster and Kalnajs (1982) velocities based on a comparison with 10 previous measurements. This correction is not confirmed at the required significance level.

The data from Mayall (1964) shows a large scatter which is well approximated by a 1- $\sigma$ uncertainty of $40 \mathrm{~km} \mathrm{~s}^{-1}$ (Table 1). This is much larger than the $25 \mathrm{~km} \mathrm{~s}^{-1}$ assigned by STPP83. After removing the systematic offset for the Minkowski (1957) data, an uncertainty of $15 \mathrm{~km} \mathrm{~s}^{-1}$ remains (as compared to $11 \mathrm{~km} \mathrm{~s}^{-1}$ assigned in STPP83). The lists by MWF88 and KFL88 are not included in Table 1 as only a few of their objects are common with $Z A S W$ data. We assign an estimated uncertainty of $15 \mathrm{~km} \mathrm{~s}^{-1}$ for all KFL88's velocities, based on the resolution of their spectra which is similar to Beaulieu's data. KFL suggest themselves an uncertainty of $8 \mathrm{~km} \mathrm{~s}^{-1}$.

\subsection{The catalogue}

Following the discussion above, we correct the sample of Minkowski (1957) for a systematic offset of $-16.3 \mathrm{~km} \mathrm{~s}^{-1}$. The offsets for other samples are not considered significant. For each $\mathrm{PN}$ the average value of its radial velocity is now obtained by weighting each existing velocity by the inverse of the square of its associated error, thus giving low weight to the poorest data:

$\bar{V}=\left[\sum_{i} \bar{V}_{i} / \operatorname{Err}_{i}^{2}\right] /\left[\sum_{i} 1 / \operatorname{Err}_{i}^{2}\right]$.

For the mean total error, we choose as the best estimate the larger of the two quantities:

$E r r=\left[\sum_{i} 1 / E r r_{i}^{2}\right]^{-\frac{1}{2}}$ 

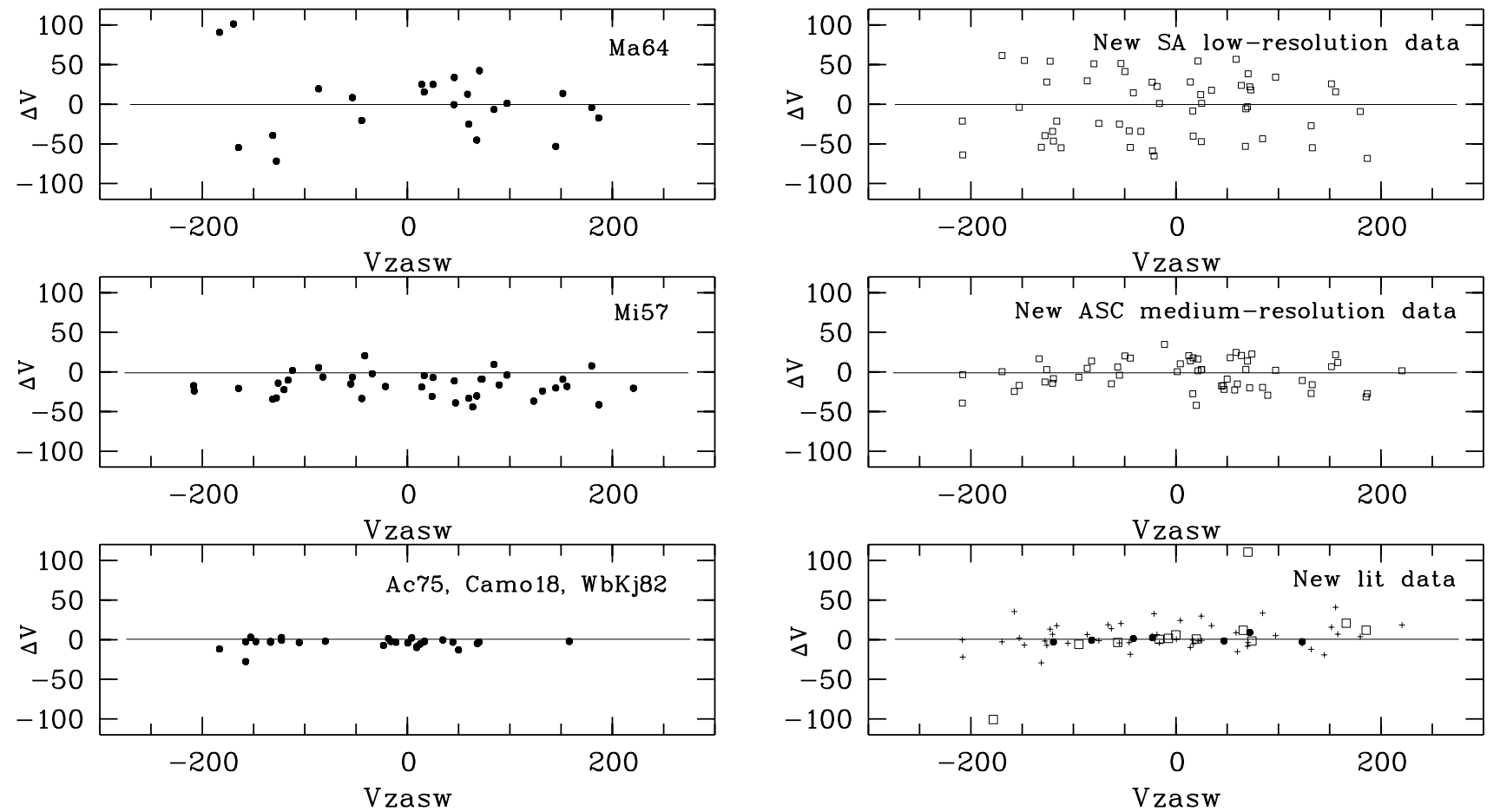

Fig. 2. Left: Comparison of the five principal sources of STPP83 with the high-dispersion $Z A S W$ data $\left(\Delta V\right.$ stands for $\left(V_{\mathrm{ZASW}}-\right.$ $\left.V_{i}\right)$ ); the literature data are globally overestimated and present various uncertainties depending from their authors. Right: Comparison of recent data with the $Z A S W$ data $\left(\Delta V\right.$ stands for $\left.\left(V_{\mathrm{ZASW}}-V_{i}\right)\right)$; in New lit data: open squares are DH97, crosses are SB96 and circles are KP95 data

and

$\operatorname{Err}_{\mathrm{rel}}=\left[\sum_{i}\left(\bar{V}_{i}-\bar{V}\right) / \operatorname{Err}_{i}^{2}\right] /\left[(P-1) \sum_{i} 1 / \operatorname{Err}_{i}^{2}\right]^{\frac{1}{2}}$

The catalogue is presented in Table 2 as follows:

Column 1: the standard PNG numbers as adopted in the SECAT.

Column 2: usual name of the PN.

Column 3: new high-resolution ZASW velocities.

Column 4: new medium-resolution ASC velocities.

Column 5: new low-resolution SA velocities.

Column 6: literature data published after 1992 with references.

Column \%: literature data from the SECAT (some are corrected from offsets seen in Table 1) with references.

Column 8: adopted radial velocities with their uncertainties.

\section{A kinematical overview of the catalogue}

\subsection{Galactic distribution}

Figure 3 (top) displays the 867 radial velocities versus galactic longitude; most $\mathrm{PNe}$ are located in the direction of the galactic Centre and characterized by highly elongated orbits. According to their galactic positions (see middle panel of Fig. 3), most PNe are concentrated towards the galactic disk, and a few $\mathrm{PNe}$ may belong to a halo population. Only BoBn 1, a very old and metaldeficient halo star (108.4-76.1, see top of Fig. 3) exhibits a completely atypical motion. Additional measurements of its velocity (the only one available was made in 1977 by Boesharr \& Bond) would be desirable in order to definitively confirm this value.

Figure 3 (bottom) displays the distribution in longitude of the $867 \mathrm{PNe}$ according to their velocity uncertainties: about $90 \%$ of the sample have velocity errors better than $20 \mathrm{~km} \mathrm{~s}^{-1}$. The largest uncertainties tend to be found among the bulge PNe.

The extrema of the radial velocities towards the galactic Centre have decreased since STPP83 paper: for example, the value of $241.0 \pm 11.0 \mathrm{~km} \mathrm{~s}^{-1}$ of M1-37 (2.6 - 3.4) in STPP83 now becomes $220.5 \pm 0.9 \mathrm{~km} \mathrm{~s}^{-1}$, and that of M4-6 (358.6 + 1.8) has gone from $-292.0 \pm 11.0 \mathrm{~km} \mathrm{~s}^{-1}$ to $-268.1 \pm 5.5 \mathrm{~km} \mathrm{~s}^{-1}$.

\subsection{The kinematics of disk $P N e$}

The use of PNe for establishing the Disk rotation curve is hampered by the large uncertainty of the distances. 


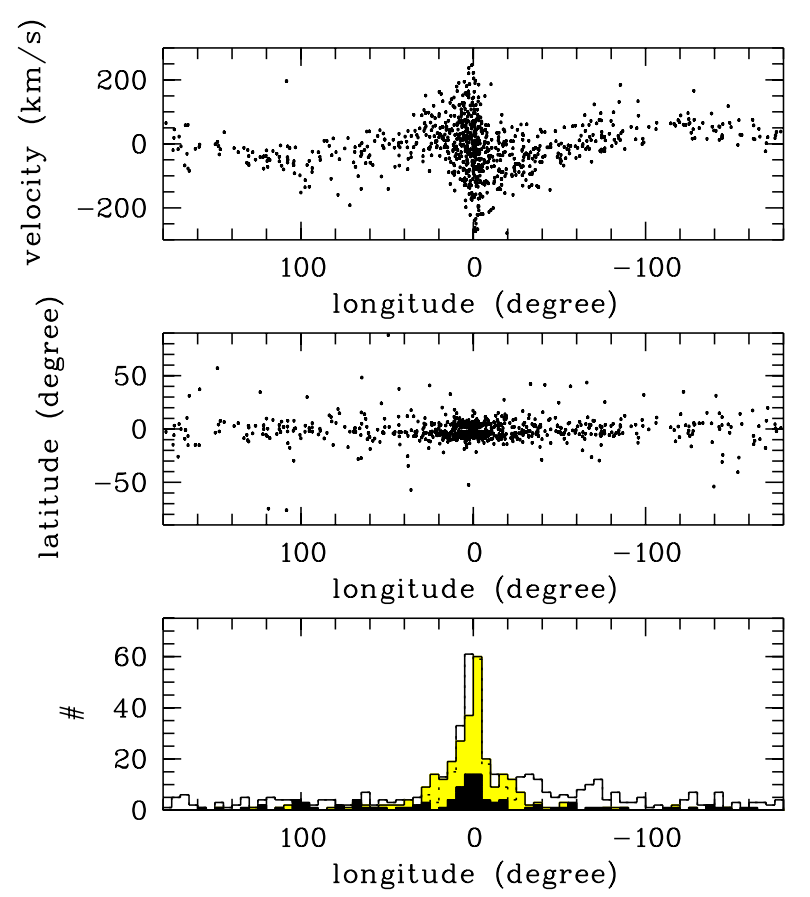

Fig. 3. Top: heliocentric radial velocities of the $867 \mathrm{PNe}$ versus their galactic longitude. Middle: galactic positions of the 867 $\mathrm{PNe}$ of the present catalogue. Bottom: distribution in longitude of the $867 \mathrm{PNe}$ according to their velocity uncertainties. White represents an error of $<10 \mathrm{~km} \mathrm{~s}^{-1}(482 \mathrm{PNe})$, grey $10 \leq$ error $<20 \mathrm{~km} \mathrm{~s}^{-1}(281 \mathrm{PNe})$, and black an error of $\geq 20 \mathrm{~km} \mathrm{~s}^{-1}$ $(104 \mathrm{PNe})$

In our study we use the statistical distance scale of Zhang (1995), which is an average of two distance scales: one is based on the correlation between the ionized mass and the radius, the other on the correlation between the radio continuum surface-brightness temperature and the nebular radius. The intrinsic uncertainty in this scale (not counting possible systematic effects) is not known but is likely to be in excess of $30 \%$ (1 sigma) for each object, from comparison with van de Steene \& Zijlstra (1994, 1995). These large uncertainties will tend to smooth out structure in the rotation curve and may also introduce systematic effects (Zijlstra \& Pottasch 1991).

We selected from our sample $100 \mathrm{PNe}$ located at $|l|>$ $7^{\circ}$ (in order to avoid contamination by bulge objects) and $<200$ pc above the galactic plane (in order to select objects with near-circular orbits). $4 \mathrm{PNe}$ with residual velocities larger than $100 \mathrm{~km} \mathrm{~s}^{-1}$ were considered interlopers and removed from the sample. Figure 4 displays the galactic distribution of the $96 \mathrm{PNe}$ projected onto the galactic plane (triangles) superimposed on all other PNe with estimated distances in the same region. Our sample seems to abruptly end at $R_{\mathrm{gc}}=5 \mathrm{kpc}$ which could be due to a local spiral arm (see for instance Georgelin \& Georgelin 1976 , for a description of the galactic spiral structure from HII regions data). Extinction by dusty molecular clouds

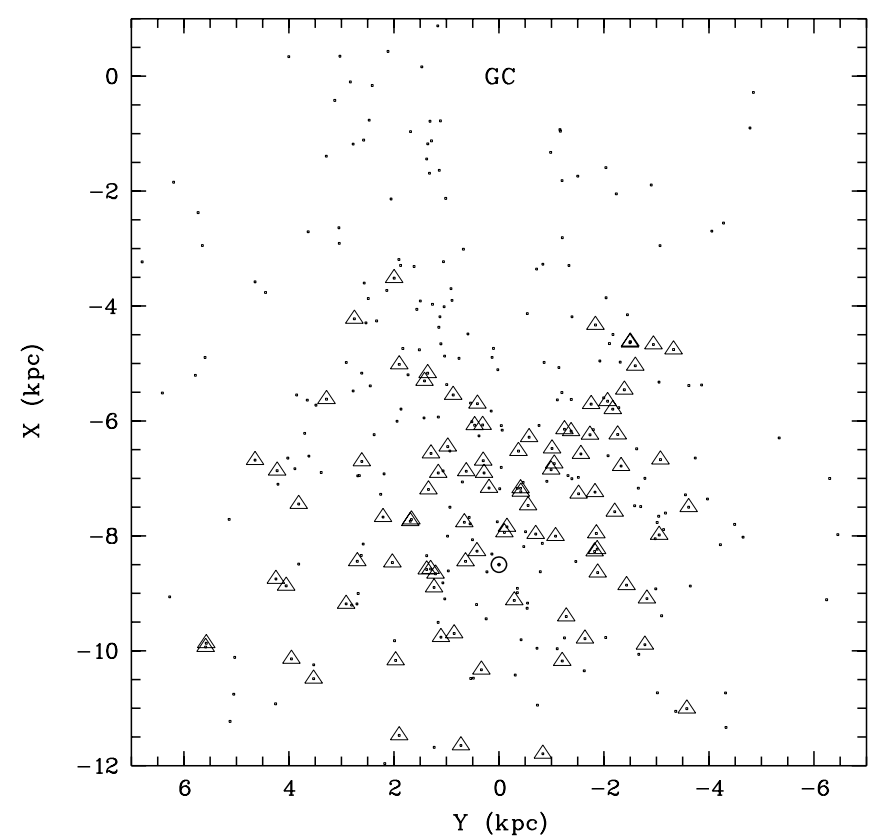

Fig. 4. Galactic distribution of the 96 disk PNe projected on the $X Y$ plane; the positions of the sun $(-8.5,0)$ and of the galactic center $(0,0)$ are indicated

in such an arm could hide farther PNe from sight.

\subsubsection{The local galactic rotation}

Consider a star on a purely circular orbit at galactocentric radius $R$ : its heliocentric radial velocity can be expressed by the well-known formula:

$V_{\mathrm{r}}^{\bmod }=-u_{\odot} \cos l \cos b-v_{\odot} \sin l \cos b-w_{\odot} \sin b$

$$
\begin{aligned}
& -2 A\left(R-R_{\odot}\right) \sin l \cos b \\
& +\left(\frac{A_{2}}{2}\left(R-R_{\odot}\right)^{2}\right) \sin l \cos b \\
& +K
\end{aligned}
$$

where:

- $u_{\odot}, v_{\odot}$ and $w_{\odot}$ are the components of the solar motion with respect to the local standard of rest, respectively in the radial, azimuthal and vertical directions $\left(u_{\odot}\right.$ is taken to be positive toward the galactic center). As our stellar sample is restricted to low vertical heights we will not be able to constrain $w_{\odot}$; instead we fix its value to the standard one $\left(7.3 \mathrm{~km} \mathrm{~s}^{-1}\right)$.

- The second row of the equation expresses the differential galactic rotation to first order; $A$ is the Oort constant.

- In the third row $A_{2}$ is the second order coefficient of the derivative of the rotation speed with respect to $R$. This expansion to second order in $\left(R-R_{\odot}\right)$ allows us to use the kinematics of stars located further from the Sun. 
Table 3. Kinematical parameters obtained from least-square fitting of Eq. (4)

\begin{tabular}{ll}
\hline$u_{\odot}$ & $16.0 \pm 4.8\left(\mathrm{~km} \mathrm{~s}^{-1}\right)$ \\
$v_{\odot}$ & $24.8 \pm 3.8\left(\mathrm{~km} \mathrm{~s}^{-1}\right)$ \\
$R_{\odot}$ & $8.7 \pm 2.1(\mathrm{kpc})$ \\
$A$ & $14.4 \pm 1.7\left(\mathrm{~km} \mathrm{~s}^{-1} \mathrm{kpc}^{-1}\right)$ \\
$A_{2}$ & $13.0 \pm 0.9\left(\mathrm{~km} \mathrm{~s}^{-1} \mathrm{kpc}^{-2}\right)$ \\
$K$ & $5.1 \pm 2.8\left(\mathrm{~km} \mathrm{~s}^{-1}\right)$ \\
\hline
\end{tabular}

- The last row gives the expression of the K-term of local galactic expansion.

We fit the above formula by minimizing:

$\chi^{2}=\sqrt{\sum_{i=1}^{N}\left(\frac{v_{\mathrm{r}}^{\mathrm{obs}_{i}}-v_{\mathrm{r}}^{\bmod _{i}}}{\Delta v_{\mathrm{r}}^{i}}\right)^{2}}$

with $N$ the total number of stars in the sample and $\Delta v_{\mathrm{r}}^{i}$ the uncertainty assigned to the radial velocity of the $i^{\text {th }}$ star.

Table 3 presents results obtained from a straightforward least-square fitting of Eq. (4).

We apply our fitting procedure by testing also various cases, as for example the non-inclusion of the $A_{2}$ term: it seems necessary to include it in the fitting procedure since without it the fits converge to unphysical values. The value of the $A_{2}$ term is largest than for other determinations (see for example Pont et al. 1994, using Cepheids data); this could be explained by the great uncertainties on $\mathrm{PNe}$ distances.

$u_{\odot}$ and $v_{\odot}$ appear different from the "standard" values (which are equal to 10.4 and $14.8 \mathrm{~km} \mathrm{~s}^{-1}$ respectively). A high value of $v_{\odot}$ is usually associated with evolved populations: it is related to the asymmetric drift which becomes more important with late-type stars (see Fig. 6 in Jahreiss $\&$ Wielen). The mean value of the asymmetric drift of our $96 \mathrm{PNe}$ sample is about $10 \mathrm{~km} \mathrm{~s}^{-1}$ in our model, but with low confidence. The high value of the $u_{\odot}$ parameter may point to the existence of an outward motion of the local standard of rest as already proposed for example by Blitz \& Spergel (1991).

The value of the $K$-term is consistent with zero given the uncertainties inherent in a multi-parameter fit. A nonzero value would be related to imperfections in the data (e.g. a systematic velocity offset or a bias) or imperfections in the rotation curve (e.g. the existence of residual nonaxisymmetrical motions). Given the uncertainty, there is no conclusive evidence for a non-zero value.

\subsubsection{The disk rotation curve}

In order to test the galactic rotation curve as function of galactocentric distance of $\mathrm{PNe}$, we first calculate the $\mathrm{PNe}$ galactic-standard-of-rest velocities using the formula:

$V_{\text {rot }}=\left(\Theta_{\odot}+\frac{V_{\mathrm{lsr}}}{\sin l \cos b}\right) \frac{R_{\mathrm{p}}}{R_{\odot}}$

where $V_{\text {lsr }}$ is calculated from the above galactic rotation model, and $\Theta_{\odot}=R_{\odot}(A-B)=8.7(14.4+12.4)=$ $233 \mathrm{~km} \mathrm{~s}^{-1}$.

In Fig. 5 we display the galactic distribution of the 96 disk PNe and the rotation velocities versus Galactocentric distance. The distance is normalized to the Solar galactocentric distance $R_{\odot}=8.7 \mathrm{kpc}$. In the bottom panel of the same figure we display the binned galactic rotation curve as provided by our PNe sample. Some authors (Amaral et al. 1996; Maciel \& Dutra 1992) have found evidence for large-scale features in their rotation curve, in particular a broad maximum near $R=6$ kpc (corresponding here to $R / R_{\odot}=0.75$ ) and a local decrease. Despite the insufficient number of objects and the (likely) smoothing due to the uncertainties in the individual distances, our curve recovers the same behaviour, but with a lower amplitude. Those features have also been detected in CO and HI data (Clemens 1985). There is also a slight indication of lower velocities around $11 \mathrm{kpc}$.

The 2 isolated points located at $R_{\mathrm{gc}}=12.05$ and $12.65 \mathrm{kpc}$ may give the appearance of an increasing outer rotation curve, but their presence cannot be in any way conclusive.

\subsection{The rotation of the bulge}

Various tracer populations have been used to constrain the structure and kinematics of the galactic bulge (Frogel et al. 1990; Minniti et al. 1992; Whitelock \& Catchpole 1992; Beaulieu 1996). These studies gave rather similar results (see also a brief review in Menzies 1990): the rotation curve increases quasi-linearly with $l$, with a mean slope of about $10-15 \mathrm{~km} \mathrm{~s}^{-1} l^{-1}$ but possibly somewhat steeper near the center. Velocity dispersions are typically $70-120 \mathrm{~km} \mathrm{~s}^{-1}$. A general trend is shown for the metal-rich populations to rotate a little faster than metal-poor ones; the velocity dispersion of the stars tends to decrease away from the Galactic Center.

KFL88 analysed a sample of $147 \mathrm{PNe}$ ranged between $|l|<10$ and $|b|<5.5$ and found indications for the rotation of the bulge: they fitted a linear equation in $\left(l, V_{\mathrm{c}}\right)$ and found the relation $V_{\mathrm{c}}=(12.0 \pm 1.9) l-(13.6 \pm 8.6) \mathrm{km} \mathrm{s}^{-1}$. They point out that due to projection effects the observed slope should be considered as lower limit.

To compare with Kinman et al. (1988), we relax our selection criteria for bulge membership: $|l|<10.0$ degree and $|b|<7.0$ degree; rejecting $\mathrm{PNe}$ with optical angular diameter $>20$ arcsec and/or radio flux $F_{6 \mathrm{~cm}}>100 \mathrm{mJy}$ (e.g. Acker \& Pottasch 1989a). This sample contains 279 

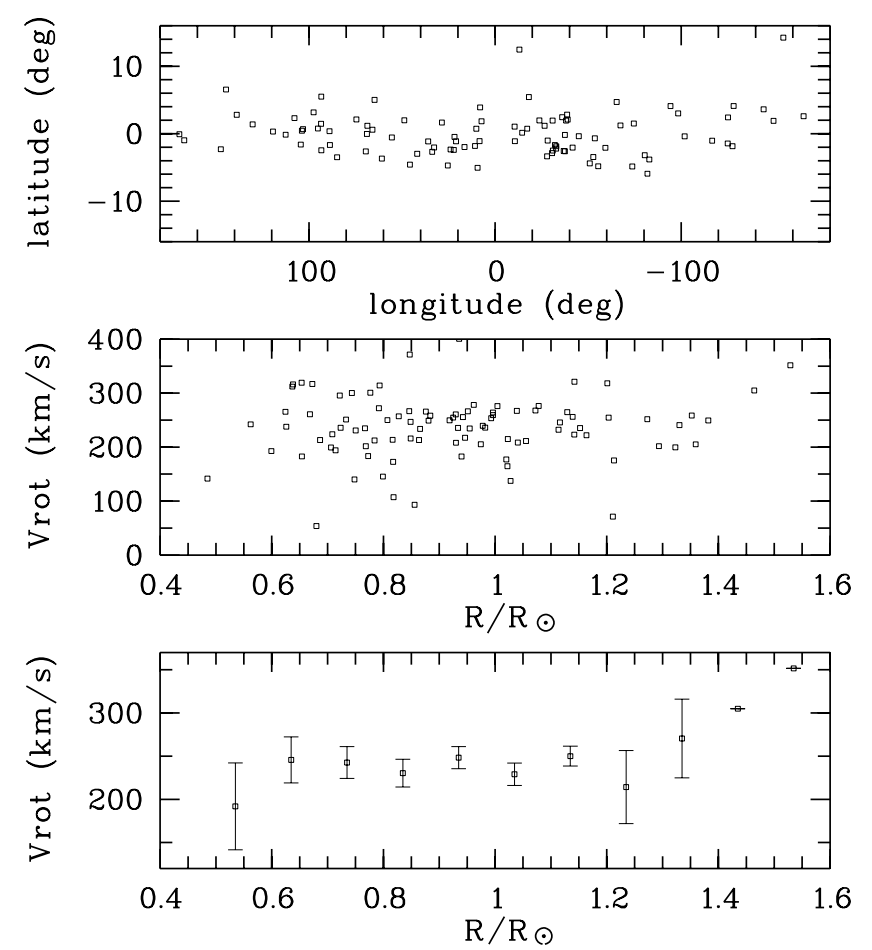

Fig. 5. Top: galactic distribution of the 96 disk PNe at $|z|<200$ pc. Middle: galactic longitude versus rotation velocity. Bottom: bin and linear fit of the middle graph. The data are averaged in 0.1 bins. The error bars include only the standard error of the mean in each bin

PNe. Figure 6 displays the galactic distribution of this PNe sample; the incompleteness is evident in the galactic plane (top panel). The middle panel shows the galacticstandard-of-rest velocities versus the longitude, and the bottom panel shows the same data in one-degree bins, with error bars representing only standard errors of the mean. The linear fit shown in the bottom of Fig. 6 has a slope of $(9.9 \pm 1.3) \mathrm{km} \mathrm{s}^{-1}$. The zero longitude offset is $-6.7 \mathrm{~km} \mathrm{~s}^{-1}$. All these results are in good agreement with KFL88.

Table 5 compares values for the bulge rotation derived from different samples. The first three lines show the results from the restricted criterion for bulge membership, using different limits for the Galactocentric distances and only velocities better than $20 \mathrm{~km} \mathrm{~s}^{-1}$. The fourth line shows the result from the relaxed criterion mentioned above. In all cases there is good evidence for the rotation of the bulge. The gradient $b$ may increase very close to the Centre but the uncertainties are much larger for this smaller samples. Interestingly, the relaxed sample gives essentially the same result. The offset at zero longitude $(a)$ is within the uncertainties zero.

The last rows of Table 5 shows for comparison previous determinations. The values are in general consistent, although the location of the tracer populations are not identical. The planetary nebulae probably provide the best
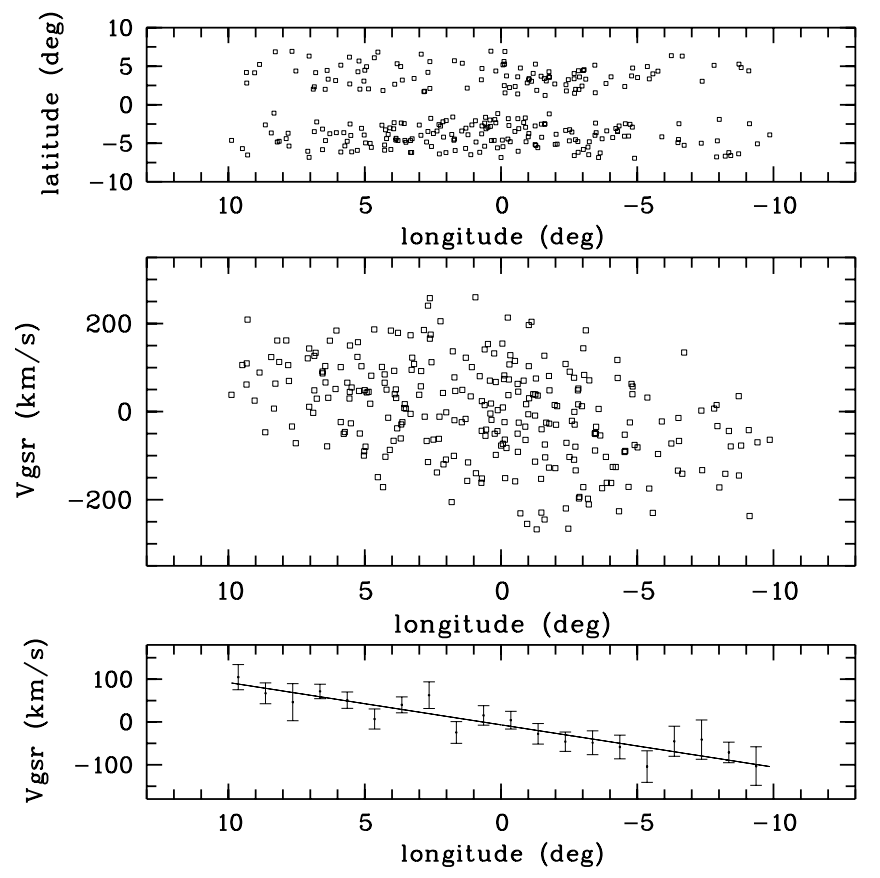

Fig. 6. Top: galactic distribution of 279 bulge PNe. The effects of the absorption in the galactic plane are evident. Middle: galactic longitude versus galactocentric velocity. Bottom: bin and linear fit of the middle graph. The data are averaged in bins of one degree of longitude. The error bars include only the standard error of the mean in each bin

coverage close to the Centre, whereas the AGB stars give better coverage at high latitudes.

\section{Conclusion}

We have presented in this paper an up-to-date compilation of all known galactic PNe radial velocities. After averaging all available data, $90 \%$ of the kinematics data of the $867 \mathrm{PNe}$ of the catalogue have accuracies better than $20 \mathrm{~km} \mathrm{~s}^{-1}$. We evaluated the kinematical parameters of the PNe differential galactic rotation by a least-square method and established the rotation curve of the Galaxy for $3.2<R<12.8 \mathrm{kpc}$. We found no significal trend for an increasing in the external rotation curve. The rotation of the bulge is found to be $(9.9 \pm 1.3) \mathrm{km} \mathrm{s}^{-1} \mathrm{deg}^{-1}$.

Acknowledgements. We are deeply indebted to Bjorn Stenholm for his participation in some of the observations. It's a great pleasure to thank Sylvie Beaulieu for generously providing us her bulge velocities before their publication. We also thank François Cuisinier for making some of his data available to us. 
Table 5. bulge rotation fitted by $a+b l \mathrm{~km} \mathrm{~s}^{-1}$

\begin{tabular}{|c|c|c|c|c|}
\hline Sample of $\mathrm{PNe}$ & Number of stars & $\begin{array}{c}a \\
\left(\mathrm{~km} \mathrm{~s}^{-1}\right)\end{array}$ & $\begin{array}{c}b \\
\left(\mathrm{~km} \mathrm{~s}^{-1} \operatorname{deg}^{-1}\right)\end{array}$ & $\begin{array}{c}\sigma \\
\left(\mathrm{kms}^{-1}\right)\end{array}$ \\
\hline$D_{\mathrm{gc}}<3 \mathrm{kpc}$ & 87 & $-9.7 \pm 11.7$ & $10.2 \pm 2.0$ & $108 \pm 12$ \\
\hline$D_{\mathrm{gc}}<2 \mathrm{kpc}$ & 60 & $-18.5 \pm 14.6$ & $12.2 \pm 3.3$ & $113 \pm 15$ \\
\hline$D_{\mathrm{gc}}<1 \mathrm{kpc}$ & 22 & $9.4 \pm 27.5$ & $32.5 \pm 12.0$ & $129 \pm 28$ \\
\hline full sample & 279 & $-6.7 \pm 8.5$ & $9.9 \pm 1.3$ & 100 \\
\hline \multicolumn{5}{|l|}{ Other populations: } \\
\hline K-giants $\left((l, b)=\left(8^{\circ}, 7^{\circ}\right)\right)($ Ref. 1$)$ & 196 & & $8.3 \pm 0.6$ & $71.9 \pm 3.6$ \\
\hline $\mathrm{SiO}$ stars $\left(b=7^{\circ}\right)($ Ref. 2$)$ & 134 & $-17.7 \pm 7.6$ & $10.8 \pm 1.2$ & 82.4 \\
\hline Miras $\left(|l|<5^{\circ}\right)$ (Ref. 3$)$ & 25 & $7.4 \pm 18$ & $22.6 \pm 8$ & $90.5 \pm 13$ \\
\hline Miras $\left(|l|<15^{\circ}, 7^{\circ}<|b|<8^{\circ}\right)($ Ref. 4$)$ & 26 & $-8.2 \pm 15.7$ & $9.8 \pm 1.9$ & $76 \pm 11$ \\
\hline
\end{tabular}

Ref. 1: Minniti 1996; 2: Izumiura et al. 1995; 3: Catchpole 1990; 4: Menzies 1990.

\section{References}

Acker A., 1975, A\&A 40, 415

Acker A., Pottasch S.R., 1989a, A\&A 221, 123

Acker A., Jasniewicz G., Köppen J., Stenholm B., 1989b, A\&AS 80, 201

Acker A., Ochsenbein F., Stenholm B., Tylenda R., Marcout J., Schon C., 1992, Strasbourg-ESO catalogue of galactic planetary nebulae, ESO (SECAT)

Acker A., Marcout J., Ochsenbein F., et al., 1996, First Supplement to the Strasbourg-ESO catalogue of Galactic Planetary Nebulae, published by the Observatoire de Strasbourg

Amaral L., Ortiz R., Lépine J., Maciel W., 1996, MNRAS 281, 347

Beaulieu S., 1996, Thesis, Australian National University

Blitz L., Spergel D., 1991, ApJ 370, 205

Boeshaar G., Bond H., 1977, ApJ 213, 421

Campbell W.W., Moore J.H., 1918, Publ. Lick Observatory 13, 75

Catchpole M., 1990, ESO/CTIO Workshop on Bulges of Galaxies, p. 111

Clemens D., 1985, ApJ 295, 422

Cuisinier F., Acker A., Köppen J., 1996, A\&A 307, 215

Dopita M.A., Hua C.T., 1997, ApJS 108, 515

Frogel J., Terndrup D., Blanco V., Whitford A., 1990, ApJ 353, 494

Georgelin Y., Georgelin P., 1976, A\&A 49, 57

Honma M., Sofue Y., 1996, PASJ 48, 103

Ibata R., Gilmore G., 1995, MNRAS 275, 605

Izumiura H., Deguchi S., Hashimoto O., et al., 1995, ApJ 453, 837

Jahreiss H., Wielen R., 1983, in the IAU Coll. 76 Nearby stars and stellar luminosity functions, p. 277

Kinman T.D., Feast M.W., Lasker B.M., 1988, AJ 95, 804 (KFL88)

Kohoutek L., Pauls R., 1995, A\&AS 111, 493

Kraan-Korteweg R., Fairall A., Woudt P., van de Steene G.C., 1996, A\&A 315, 549

Lewis J., Freeman K., 1989, AJ 97, 139
Le Bertre T., Epchtein N., Gouiffes C., Heydari-Malayeri M., Perrier C., 1989, A\&A 225, 417

Maciel W., Dutra C., 1992, A\&A 262, 271

Mayall N.U., 1964 (priv. comm. in: Perek \& Kohoutek 1967)

Meatheringham S., Wood P., Faulkner D., 1988, ApJ 334, 862 (MWF88)

Menzies J., 1990, ESO/CTIO Workshop on Bulges of Galaxies, p. 115

Menzies J.W., Wolstencroft R.D., 1990, MNRAS 247, 177

Minkowski D.K., 1957 (priv. comm. in: Perek \& Kohoutek 1967)

Minniti D., White S., Olszewski E., Hill J., 1992, ApJ 393, L47

Minniti D., 1994, PASP 106, 813

Minniti D., 1996, ApJ 459, 579

Morrison H., Flynn C., Freeman K., 1990, AJ 100 (4), 1191

Peimbert M., 1992, in: Planetary Nebulae, IAU Symp. 180, Weinberger R. and Acker A. (eds.). Kluwer, Dordrecht, p. 523

Perek L., Kohoutek L., 1967, catalogue of galactic planetary nebulae, Czechoslovak Institute of Science, Prague

Pont F., Mayor M., Burki G., 1994, A\&A 285, 415

Schneider S.E., Terzian Y., Purgathofer A., Perinotto M., 1983a, ApJS 52, 399 (STPP83)

Schneider S.E., Terzian Y., 1983b, ApJ 274, L61

Sevenster M.N., Chapman J.M., Habing H.J., Killeen N.E.B., Lindquist M., 1997, A\&AS 122, 79

Spyromilio J., 1995, MNRAS 277, L59

van de Steene G.C., Zijlstra A.A., 1994, A\&AS 108, 485

van de Steene G.C., Zijlstra A.A., 1995, A\&A 293, 541

Webster B.L., Kalnajs A., 1982 (priv. comm. in Schneider et al. 1983a)

Whitelock P., Catchpole R., 1992, in: The center, Bulge, and Disk of the Milky Way, L. Blitz (ed.). Kluwer Academic Publishers, p. 103

Zhang C., 1995, ApJS 98, 659

Zhao H., Spergel D., Rich M., 1994, AJ 108, 2154

Zijlstra A.A., Pottasch S.R., 1991, A\&A 243, 478

Zijlstra A.A., Walsh J., 1996, A\&A 312, L21

Zijlstra A.A., Acker A., Walsh J., 1997, A\&AS 125, 289 